\title{
Application of the Flow Curvature Method in Lorenz-Haken Model
}

\author{
A. K. M. Nazimuddin ${ }^{\text {a,* }}$, Md. Showkat Ali ${ }^{\mathrm{b}}$ \\ ${ }^{a}$ Department of Mathematical and Physical Sciences, East West University, Dhaka-1212, Bangladesh \\ ${ }^{b}$ Department of Applied Mathematics, University of Dhaka, Dhaka-1000, Bangladesh
}

Received: 31 May 2019; Accepted: 14 June 2019; Published: 08 February 2020

\begin{abstract}
We consider a recently developed new approach so-called the flow curvature method based on the differential geometry to analyze the Lorenz-Haken model. According to this method, the trajectory curve or flow of any dynamical system of dimension $n$ considers as a curve in Euclidean space of dimension $n$. Then the flow curvature or the curvature of the trajectory curve may be computed analytically. The set of points where the flow curvature is null or empty defines the flow curvature manifold. This manifold connected with the dynamical system of any dimension $n$ directly describes the analytical equation of the slow invariant manifold incorporated with the same dynamical system. In this article, we apply the flow curvature method for the first time on the three-dimensional Lorenz-Haken model to compute the analytical equation of the slow invariant manifold where we use the Darboux theorem to prove the invariance property of the slow manifold. After that, we determine the osculating plane of the dynamical system and find the relation between flow curvature manifold and osculating plane. Finally, we find the nature of the fixed point stability using flow curvature manifold.
\end{abstract}

Index Terms: Flow Curvature Method, Slow Manifold, Dynamical System, Differential Geometry.

(C) 2020 Published by MECS Publisher. Selection and/or peer review under responsibility of the Research Association of Modern Education and Computer Science

\section{Introduction}

According to [1, 2, 3] singularly perturbed systems can have invariant manifolds where the trajectories of the flow moves slowly and these slow manifolds are invariant with respect to the flow. Several methods have been developed to find out the analytical slow manifold equations of the singularly perturbed systems. In [4, 5 , $6,7,8,9,10,11]$, introduces the geometric singular perturbation technique to establish the existence of the slow manifold equation along with the local invariance of the slow manifold for the singularly perturbed system. In the case of non-singularly perturbed system like that of L-H model this technique fails to provide the slow manifold.

* Corresponding author.

E-mail address: 
The flow curvature method $[12,13,14,15,16]$ is the new method in recent publications for computing the analytical implicit equation of the slow manifold. This method can be applied to any autonomous or nonautonomous dynamical systems in $\mathrm{n}$ dimensions whether it is singularly perturbed or not. Recent applications of the flow curvature method of the singularly perturbed systems are FitzHugh-Nagumo model, Brusselator model, Van der pol model, Chua's model, etc and the applications of the flow curvature method of the nonsingularly perturbed systems are Lorenz model, Rikitake model, etc. [17] used the flow curvature method to construct the slow invariant manifold of the heartbeat model. In [18], author developed the slow invariant manifold analytical implicit equation of the generalized Lorenz-Krishnamurthy model and conservative generalized Lorenz-Krishnamurthy model. The most important feature of this method is that, the flow curvature manifold directly gives us the analytical equation of the slow manifold. Without using any asymptotic expansions, this method allows us to find the flow curvature manifold and hence slow invariant manifold equation. To the best of our knowledge, this method is the best to find the analytical equation of the slow invariant manifold for any dimensional dynamical system and this study advances the field from the previous related work.

Several attempts have been taken to compute the slow manifold equation of the Lorenz-Haken (L-H) model. In [19], an Iterative approach is studied where the implicit equation of the slow manifold of the L-H model is calculated. Then [20] proposed a new technique to compute the equation of the slow manifold of the L-H model and this method is based on the slow eigenvectors of the local tangent systems. [21] identified the slow manifold of the L-H model by using the geometric singular perturbation theory. So, our goal of this present paper is to apply the flow curvature method on the three dimensional laser system called L-H model to find the analytical implicit equation of the slow invariant manifold. The invariance of the slow manifold of the L-H model is then proved by using the Darboux theory. We also find osculating plane of the L-H model where osculating plane passes through the fixed points of the dynamical system. We discuss also the nature of the fixed points stability by using the flow curvature manifold. To simulate the L-H model, we use MATHEMATICA as a software tool.

The remainder part of this paper is organized as follows. In section 2, we describe the nonlinear optical slowfast L-H model. We discuss the flow curvature method based on the differential geometry in section 3 . We also present the Darboux theorem in this section which represents the invariance condition of the slow manifold of a dynamical system. In section 4, we express the slow manifold analytical equation of the L-H model. Osculating plane equations of the $\mathrm{L}-\mathrm{H}$ model is also determined in section 5. In section 6, we discussed about the fixed points stability using flow curvature manifold. Some conclusions are given in section 7.

\section{L-H Model}

In [22], Haken introduced an optical model. Since the Haken model is similar to the Lorenz model, hence the system is called L-H model. The slow-fast nonlinear system of equations in three variables for the standard L-H model is given by:

$$
\begin{aligned}
& \dot{E}=\bar{k}(P-E), \\
& \dot{P}=n E-P, \\
& \dot{n}=\bar{\gamma}(B-n-E P) .
\end{aligned}
$$

In the laser system (1), the real amplitude of the electromagnetic field is denoted by $E$, the polarization of the cavity medium is denoted by $P$ and $n$ is the inversion of the state within the two levels of the development due to the pumping. Also, $\bar{k}$ and $\bar{\gamma}$ are the relaxation rate parameters and $B$ is the pump parameter. If we consider $x, y, z$ in place of $E, P, n$ respectively and also consider $\mu, \delta$ in place of $\bar{k}, \bar{\gamma}$, then equation (1) can be written as the following system of non-linear ordinary differential equations. 


$$
\begin{aligned}
& \dot{x}=\mu(y-x), \\
& \dot{y}=z x-y, \\
& \dot{z}=\delta(B-z-x y) .
\end{aligned}
$$

\section{Flow Curvature Method}

In this part, we briefly discuss the flow curvature method in terms of differential geometry. This method uses the properties of curvatures of trajectory curve or flow of the dynamical system. Using this method, one can define the flow curvature manifold corresponding to the dynamical system. Any n-dimensional dynamical system can have the (n-1) dimensional flow curvature manifold that means flow curvature manifold contains the information about the flow with highest curvature.

\subsection{Analytical Implicit Equation of the Slow Manifold of the Dynamical System}

Invariant manifold implies a very significant role to explain the stability as well as dynamical behavior of a system, especially for a slow-fast dynamical system. Although geometric perturbation technique is well known to find the analytical equation of slow manifold, the main difference between geometric perturbation technique and the flow curvature method is that it neither uses asymptotic expansions nor eigenvectors. Another difference is that this method can be used for any dynamical system which may or may not singularly perturbed.

Proposition 3.1 The set of points where the curvature of the flow of the model (2) vanishes represented by the following flow curvature manifold equation of the dynamical system.

$$
\psi(\vec{X})=\overrightarrow{\dot{X}} \cdot(\overrightarrow{\ddot{X}} \wedge \ddot{X})=\operatorname{det}(\overrightarrow{\dot{X}}, \vec{X}, \ddot{X})=0
$$

Proof See $[13,14]$

Note that for any n-dimensional dynamical system, maximum $(\mathrm{n}-1)^{\text {th }}$ flow curvature is possible.

Proposition 3.2 The flow curvature manifold of the dynamical system (2) directly provides its implicit analytical equation of the slow manifold.

Proof See $[13,14]$

\subsection{Darboux Invariance Theorem}

According to [23, 24], the concept of the invariant manifold is first introduced by G. Darboux (1878, p. 71). We consider the trajectories of the dynamical system (2) is represented by a motion of a point in a three dimensional space and the coordinates of the point is $\vec{X}=(x, y, z)$ and the velocity vector of this point is $\vec{V}=(\dot{x}, \dot{y}, \dot{z})$.

Proposition 3.3 Consider $\psi(\vec{X})=\operatorname{det}(\vec{X}, \overrightarrow{\dot{X}}, \vec{X})=0$ is a slow manifold of the dynamical system (2) where $\psi$ is a first time continuously differentiable function, then this manifold is invariant with respect to the flow of (2) if there exist a first time continuously differentiable function called cofactor $C(\vec{X})$ which satisfies the following equation:

$$
\mathrm{L}_{\vec{V}} \psi(\vec{X})=C(\vec{X}) \psi(\vec{X})
$$


with the Lie derivative defined as the following:

Proof See [13, 14]

$$
\mathrm{L}_{\vec{V}} \psi(\vec{X})=\vec{V} \cdot \vec{\nabla} \psi=d \psi / d t
$$

\subsection{The Osculating Plane Equation}

Definition 3.1 The osculating plane is the plane which passes through a fixed point $\vec{X}^{*}$ of the dynamical system and parallel to the unit tangent and normal vectors to a three dimensional curve and also spanned by the velocity vector and acceleration vector. The Osculating plane can be defined using for a dynamical system (2) as the following

$$
P(\vec{X})=\left(\vec{X}-\vec{X}^{*}\right) \cdot(\vec{X} \wedge \vec{X})=0
$$

Theorem 3.4 The Flow curvature manifold $\psi(\vec{X})$ of the three-dimensional dynamical system (2) merges with its Lie derivative $L_{\vec{V}} \psi(\vec{X})$ and with its osculating plane $P(\vec{X})$ in the vicinity of the fixed point $\vec{X}^{*}$.

Proof See [14]

\subsection{Stability Analysis of the Fixed Points}

Definition 3.2 The fixed points $\vec{X}^{*}$ of any dynamical system may also be fixed points of the flow curvature manifold if the following two equations are satisfied:

$$
\begin{aligned}
& \psi\left(\vec{X}^{*}\right)=0 \\
& \vec{\nabla} \psi\left(\vec{X}^{*}\right)=0
\end{aligned}
$$

Definition 3.3 The Hessian of a function $\psi(\vec{X})$ at the point $\vec{X}$ is denoted by $H_{\psi(\vec{X})}$ and defined by

$$
H_{\psi(\vec{X})}=\left|\begin{array}{lll}
\frac{\partial^{2} \psi}{\partial x^{2}} & \frac{\partial^{2} \psi}{\partial x \partial y} & \frac{\partial^{2} \psi}{\partial x \partial z} \\
\frac{\partial^{2} \psi}{\partial y \partial x} & \frac{\partial^{2} \psi}{\partial y^{2}} & \frac{\partial^{2} \psi}{\partial y \partial z} \\
\frac{\partial^{2} \psi}{\partial z \partial x} & \frac{\partial^{2} \psi}{\partial z \partial y} & \frac{\partial^{2} \psi}{\partial z^{2}}
\end{array}\right|
$$

Theorem 3.5 The relative Hessian of the flow curvature manifold $\psi(\vec{X})$ of the three-dimensional dynamical system (2) enables to determine the stability of the fixed point $\vec{X}^{*}$.

If $\hat{H}_{\psi\left(\vec{X}^{*}\right)} \geq 0$ then both eigenvalues are real and the fixed point $\vec{X}^{*}$ is a saddle-node.

If $\hat{H}_{\psi\left(\vec{X}^{*}\right)}<0$ then eigenvalues are complex conjugated and the fixed point $\vec{X}^{*}$ is a saddle-focus or a center. 
Proof See [14]

\section{The Slow Invariant Manifold of the L-H model}

According to the flow curvature method, the trajectory curves of any dynamical system which may or may not singularly perturbed considered the curves in the Euclidean space. We consider the system model (2) as the slow-fast dynamical system.

We use the parameter values of (2) as mentioned in Table 1 and for the numerical simulation, we consider the range of the state variables connected with the dynamical system (2) as the following

$$
\begin{aligned}
& {\left[\mathrm{x}_{\min }, \mathrm{x}_{\max }\right]=[-4,4] ;} \\
& {\left[\mathrm{y}_{\min }, \mathrm{y}_{\max }\right]=[-4,4] ;} \\
& {\left[\mathrm{z}_{\min }, \mathrm{z}_{\max }\right]=[0,15] ;}
\end{aligned}
$$

TABLE 1. TYPICAL PARAMETER VALUES OF (2) FOR THE NUMERICAL COMPUTATIONS.

\begin{tabular}{llcc}
\hline Parameters & $\mu$ & $\delta$ & $\mathrm{B}$ \\
\hline Values & 4.0 & 0.4 & 12.0 \\
\hline
\end{tabular}

By putting right hand side parts of the dynamical system (2), that is,

$$
\begin{aligned}
& \mu(y-x)=0, \\
& y z x-y=0, \\
& \delta(B-z-x y)=0,
\end{aligned}
$$

we obtain three following graphs for the three null-clines of the system (2).

Thus, we get the following three fixed points by solving the system (3)

$$
\begin{aligned}
& \mathrm{x}_{1}=-3.3166247903554 ; \mathrm{y}_{1}=-3.3166247903554 ; \mathrm{z}_{1}=1 ; \\
& \mathrm{x}_{2}=0 ; \mathrm{y}_{2}=0 ; \mathrm{z}_{2}=12 ; \\
& \mathrm{x}_{3}=3.31662479103554 ; \mathrm{y}_{3}=3.3166247903554 ; \mathrm{z}_{3}=1 ;
\end{aligned}
$$

We use explicit Runge-Kutta method to solve the model (2) numerically where we use $\left(x_{0}, y_{0}, z_{0}\right)=(1,1,1)$ as an initial point. Fig.1 shows the phase diagram represented by (2) where $t$ ranges from 500 to 1000 . Also, the three green points in the fig. 1 indicate the fixed points of the model (2). 


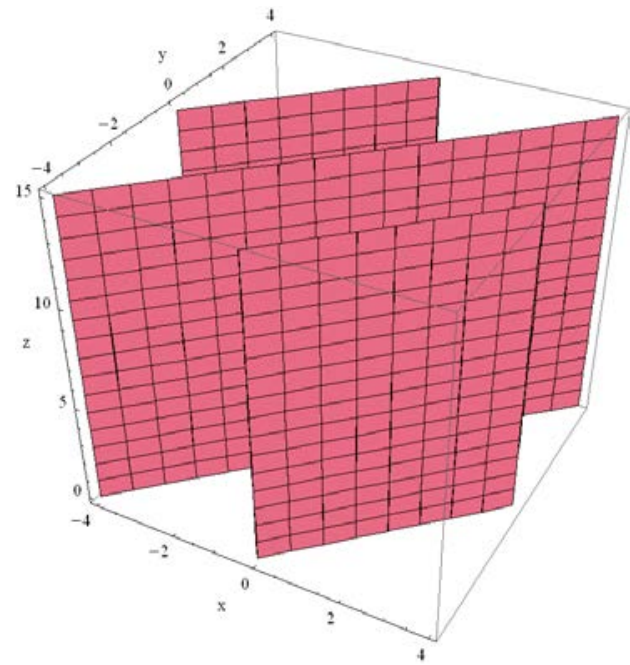

(a)

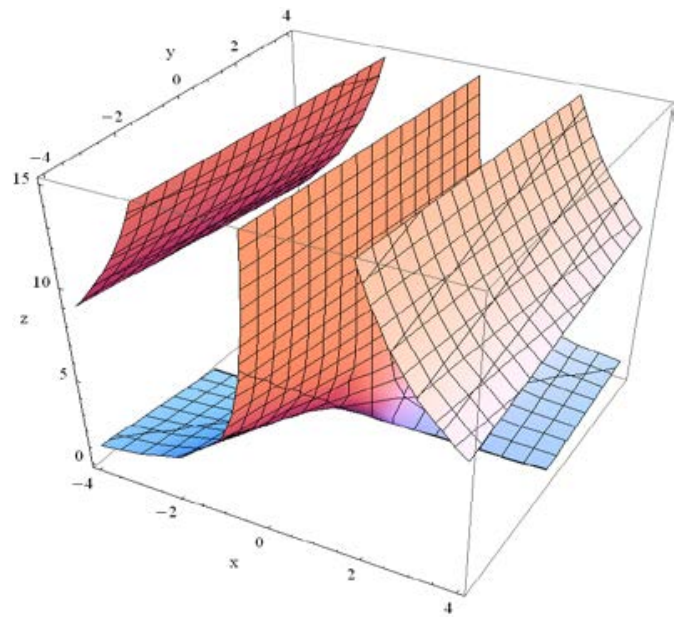

(b)

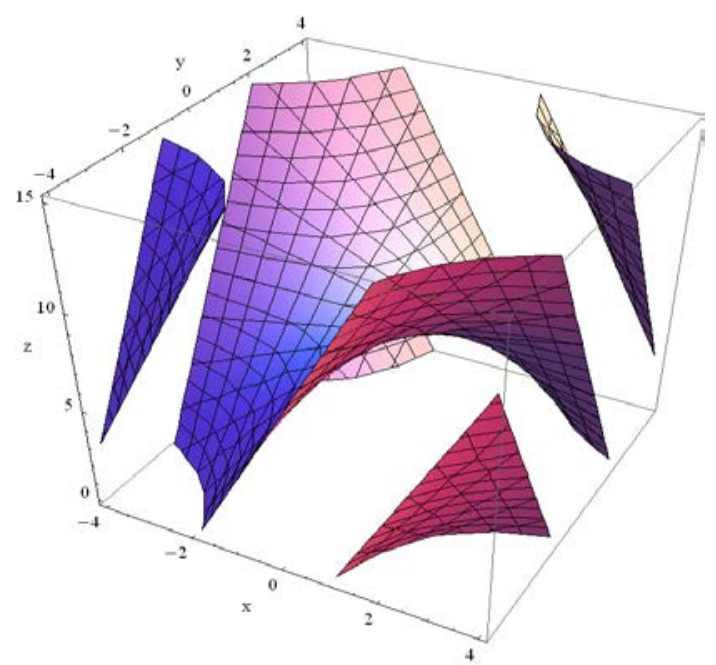

(c)

Fig.1. Nullclines of the model (2). (a) first equation of the system (3), (b) second equation of the system (3) and (c) third equation of the system (3).

Thus, we get the following three fixed points by solving the system (3)

$$
\begin{aligned}
& \mathrm{x}_{1}=-3.3166247903554 ; \mathrm{y}_{1}=-3.3166247903554 ; \mathrm{z}_{1}=1 ; \\
& \mathrm{x}_{2}=0 ; \mathrm{y}_{2}=0 ; \mathrm{z}_{2}=12 ; \\
& \mathrm{x}_{3}=3.31662479103554 ; \mathrm{y}_{3}=3.3166247903554 ; \mathrm{z}_{3}=1 ;
\end{aligned}
$$


We use explicit Runge-Kutta method to solve the model (2) numerically where we use $\left(x_{0}, y_{0}, z_{0}\right)=(1,1,1)$ as an initial point. Fig.1 shows the phase diagram represented by (2) where $t$ ranges from 500 to 1000 . Also, the three green points in the fig. 1 indicate the fixed points of the model (2).

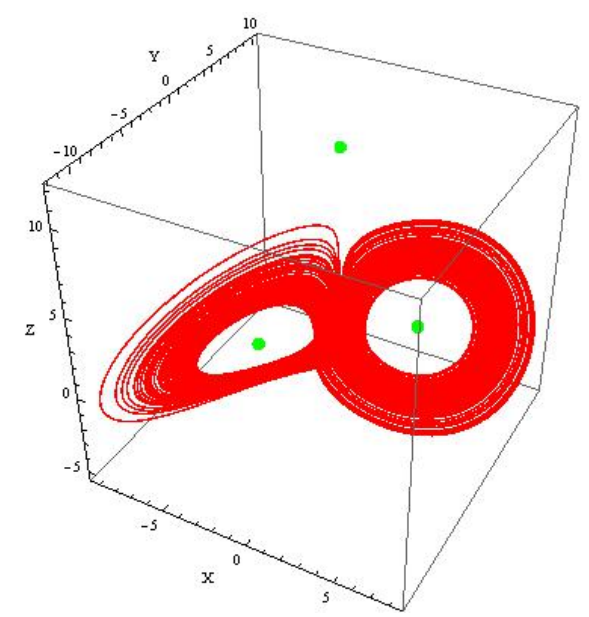

Fig.2. Phase plot analysis for the model (2) along with the three fixed points obtained from the same model.

Now, in order to calculate the flow curvature manifold of the model (2) using the flow curvature method, we need the velocity, acceleration and over-acceleration (Jerk) because of our 3-dimensional dynamical model. The velocity vector field of the model (2) can be represented by the following way.

$$
\vec{V}=\{4(-x+y),-y+x z, 0.4(12-x y-z)\}
$$

The Jacobian matrix corresponding to the model (2) may be written as

$$
J=\left(\begin{array}{ccc}
-4 & 4 & 0 \\
z & -1 & x \\
-0.4 y & -0.4 x & -0.4
\end{array}\right)
$$

Now we get the acceleration vector by using the formula $\vec{A}=J \vec{V}$ and hence, we obtain

$$
\vec{A}=4(4 x-5 y+x z),-0.4\left(-12 x-2.5 y+x^{2} y+13.5 x z-10 y z\right),-1.6\left(1.2-1.35 x y+y^{2}-0.1 z+0.25 x^{2} z\right)
$$

Then, the over-acceleration or jerk is calculated according to the formula $\overrightarrow{A^{*}}=J \vec{A}+$ TotalDifferential $(J) \vec{V}$ and we get the result as the following.

$$
\begin{aligned}
& \overrightarrow{A^{*}}=-1.6\left(-12 x-2.5 y+x^{2} y+13.5 x z-10 y z\right), 3.2(-x+y)(12-x y-z)+4 z(4 x-5 y+x z)- \\
& 1.6 x\left(1.2-1.35 x y+y^{2}-0.1 z+0.25 x^{2} z\right)+0.4\left(-12 x-2.5 y+x^{2} y+13.5 x z-10 y z\right),- \\
& 1.6 y(4 x-5 y+x z)+0.64\left(1.2-1.35 x y+y^{2}-0.1 z+0.25 x^{2} z\right)+0.16 x\left(-12 x-2.5 y+x^{2} y+13.5 x z-10 y z\right)
\end{aligned}
$$


After that, we find the slow manifold function of the model (2) as the following

$$
\psi(x, y, z)=0.256\left(\begin{array}{l}
-8208 x^{2}+144 x^{4}+18702 x y+1218 x^{3} y-24 x^{5} y-13896 y^{2}-2226 x^{2} y^{2}+ \\
18 x^{4} y^{2}+x^{6} y^{2}+764 x y^{3}-110 x^{3} y^{3}+720 y^{4}+192 x^{2} y^{4}-120 x y^{5}+ \\
3186 x^{2} z-282 x^{4} z-4462.5 x y z+672 x^{3} y z-1.5 x^{5} y z+4110 y^{2} z- \\
718 x^{2} y^{2} z-20 x^{4} y^{2} z-24 x y^{3} z+30 x^{3} y^{3} z+160 y^{4} z-808.5 x^{2} z^{2}+45 x^{4} z^{2}+ \\
2.5 x^{6} z^{2}+1142 x y z^{2}-260 x^{3} y z^{2}-1446 y^{2} z^{2}+425 x^{2} y^{2} z^{2}-300 x y^{3} z^{2}+ \\
350 x^{2} z^{3}-50 x^{4} z^{3}-75 x y z^{3}+75 x^{3} y z^{3}+100 . y^{2} z^{3}-25 x^{2} z^{4}
\end{array}\right)
$$

Now the analytical implicit equation of the slow manifold of the model (2) can be written as

$$
\psi(x, y, z)=0
$$

In Fig.3(a) shows the graphical representation of the analytical implicit equation of the slow manifold represented by the equation (4) and Fig.3(b) represents the slow manifold as well as phase space diagram in the same graph.

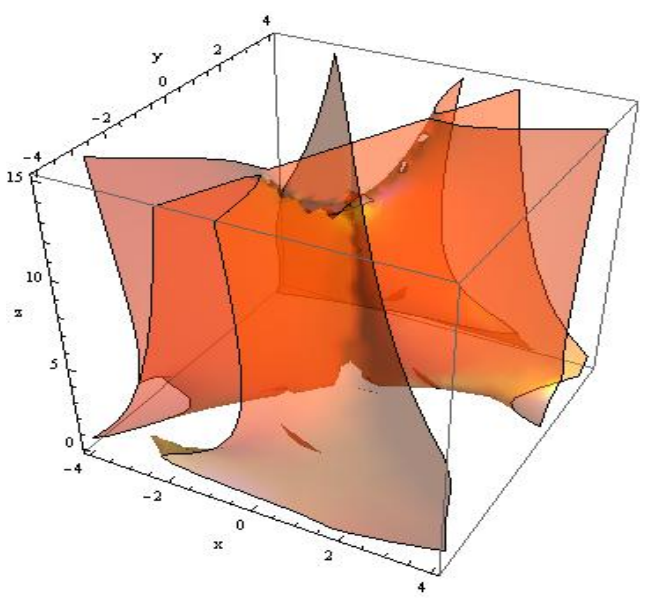

(a)

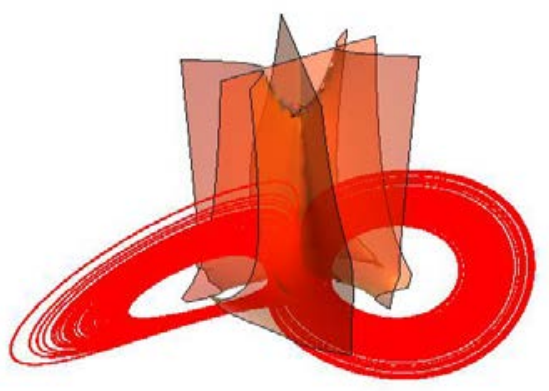

(b)

Fig.3. (a) Graphical representation of the slow manifold analytical equation of the model (2) using flow curvature method, (b) Graphical representation of the slow manifold analytical equation along with the phase diagram represented by (2).

The Lie derivative of the slow manifold function is then evaluated as the following by using the Darboux invariance theory to establish the flow curvature invariance of the equation (4). We first find the normal vector of the flow curvature manifold and we get 


$$
\begin{aligned}
& \vec{\nabla} \psi=\left\{0 . 2 5 6 \left(-16416 . x+576 . x^{3}+18702 \cdot y+3654 . x^{2} y-120 . x^{4} y-4452 . x y^{2}+\right.\right. \\
& 72 . x^{3} y^{2}+6 \cdot x^{5} y^{2}+764 \cdot y^{3}-330 \cdot x^{2} y^{3}+384 . x y^{4}-120 \cdot y^{5}+6372 \cdot x z-1128 \cdot x^{3} z- \\
& 4462.5 y z+2016 . x^{2} y z-7.5 x^{4} y z-1436 . x y^{2} z-80 . x^{3} y^{2} z-24 . y^{3} z+90 . x^{2} y^{3} z- \\
& \text { 1617.xz } z^{2}+180 \cdot x^{3} z^{2}+15 \cdot x^{5} z^{2}+1142 \cdot y z^{2}-780 \cdot x^{2} y z^{2}+850 \cdot x y^{2} z^{2}-300 \cdot y^{3} z^{2}+ \\
& \text { 700.xz } \left.z^{3}-200 \cdot x^{3} z^{3}-75 \cdot y z^{3}+225 \cdot x^{2} y z^{3}-50 . x z^{4}\right), 0.256\left(18702 . x+1218 \cdot x^{3}-\right. \\
& 24 . x^{5}-27792 \cdot y-4452 \cdot x^{2} y+36 \cdot x^{4} y+2 \cdot x^{6} y+2292 \cdot x y^{2}-330 \cdot x^{3} y^{2}+2880 \cdot y^{3}+ \\
& 768 . x^{2} y^{3}-600 . x y^{4}-4462.5 x z+672 . x^{3} z-1.5 x^{5} z+8220 . y z-1436 . x^{2} y z-40 . x^{4} y z- \\
& 72 . x y^{2} z+90 \cdot x^{3} y^{2} z+640 \cdot y^{3} z+1142 \cdot x z^{2}-260 \cdot x^{3} z^{2}-2892 \cdot y z^{2}+850 \cdot x^{2} y z^{2}- \\
& \left.900 . x y^{2} z^{2}-75 \cdot x z^{3}+75 \cdot x^{3} z^{3}+200 \cdot y z^{3}\right), 0.256\left(3186 . x^{2}-282 \cdot x^{4}-4462.5 x y+\right. \\
& 672 . x^{3} y-1.5 x^{5} y+4110 . y^{2}-718 . x^{2} y^{2}-20 . x^{4} y^{2}-24 . x y^{3}+30 . x^{3} y^{3}+160 \cdot y^{4}- \\
& \text { 1617. } x^{2} z+90 \cdot x^{4} z+5 \cdot x^{6} z+2284 \cdot x y z-520 \cdot x^{3} y z-2892 \cdot y^{2} z+850 \cdot x^{2} y^{2} z-600 \cdot x y^{3} z+ \\
& \text { 1050. } \left.\left.x^{2} z^{2}-150 \cdot x^{4} z^{2}-225 x y z^{2}+225 x^{3} y z^{2}+300 y^{2} z^{2}-100 x^{2} z^{3}\right)\right\}
\end{aligned}
$$

Now according to proposition 3.3, we compute Lie derivative of the slow manifold as follows

$$
\mathrm{L}_{\bar{v}} \psi=-6.5024\left(\begin{array}{l}
-3187.28 x^{2}+144 . x^{4}+7110 x y+455.858 x^{3} y-24 x^{5} y-4816.06 y^{2}-1386.4 x^{2} y^{2}+ \\
46.0157 x^{4} y^{2}+x^{6} y^{2}+980.913 x y^{3}-93.2756 x^{3} y^{3}-1.25984 x^{5} y^{3}-37.1654 y^{4}+ \\
142.299 x^{2} y^{4}+0.472441 x^{4} y^{4}-100.472 x y^{5}+18.8976 y^{6}+622.913 x^{2} z-247.039 x^{4} z- \\
1289.63 x y z+780.236 x^{3} y z-1.26378 x^{5} y z+1637.62 y^{2} z-826.362 x^{2} y^{2} z- \\
8.50394 x^{4} y^{2} z+173.606 x y^{3} z+13.937 x^{3} y^{3} z+31.4961 y^{4} z-4.37096 \times 10^{-15} x^{2} y^{4} z- \\
302.846 x^{2} z^{2}+31.6535 x^{4} z^{2}+2.5 x^{6} z^{2}+234.315 x y z^{2}-139.055 x^{3} y z^{2}-3.14961 x^{5} y z^{2}- \\
395.937 y^{2} z^{2}+302.835 x^{2} y^{2} z^{2}-246.457 x y^{3} z^{2}+47.2441 y^{4} z^{2}+100.709 x^{2} z^{3}-23.622 x^{4} z^{3}- \\
14.685 x y z^{3}+38.3858 x^{3} y z^{3}+24.4094 y^{2} z^{3}-6.49606 x^{2} z^{4}-2.95276 x^{4} z^{4}-1.09274 \times 10^{-15} x y z^{4}
\end{array}\right)
$$

In Fig.4(a) shows the graphical representation of the equation $\mathrm{L}_{\vec{v}} \psi=0$ that means the graphical representation of the flow curvature invariance manifold where the rate of change of $\psi(x, y, z)$ is equal to zero and Fig.4(b) shows the combined graph of the invariance manifold and phase space plot. 


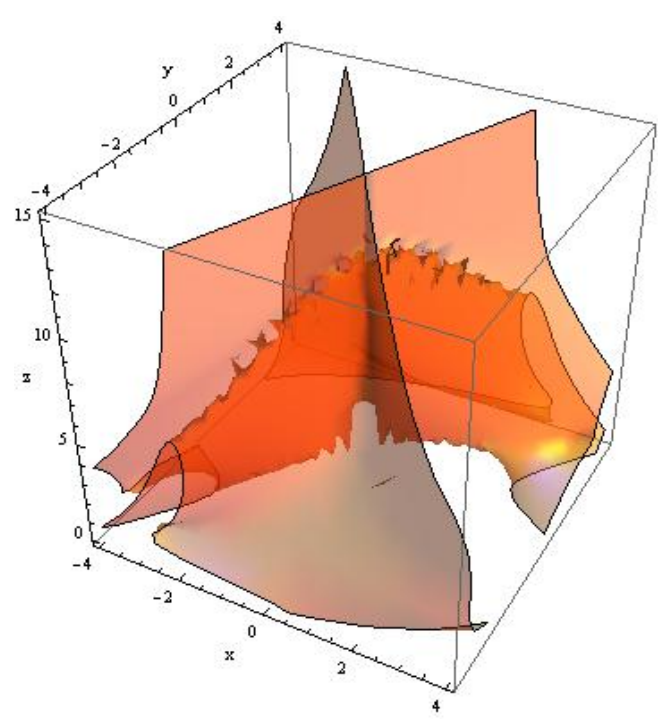

(a)

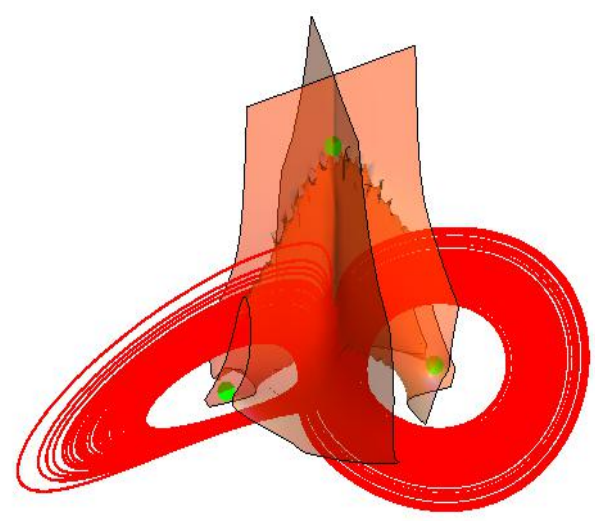

(b)

Fig.4. (a) Graphical representation of the invariance equation of the slow manifold analytical equation of the model (2) according to the Darboux theorem (b) Graphical representation of the invariance equation of the slow manifold analytical equation along with the phase diagram represented by (2).

\section{The Osculating Plane Equation of the L-H Model}

According to the definition 3.1, we calculate the osculating plane equation for the fixed point $\mathrm{x}_{1}=-3.3166247903554, \mathrm{y}_{1}=-3.3166247903554, \mathrm{z}_{1}=1$. We find the first osculating plane expression as the following

$$
P_{1}(\vec{X})=6.4\left(\begin{array}{l}
23.8797 x-0.6 x^{2}-47.2619 y+5.475 x y+3.15079 x^{2} y+0.35 x^{3} y-11.3 y^{2}- \\
4.56036 x y^{2}+0.325 x^{2} y^{2}-0.0829156 x^{3} y^{2}-0.025 x^{4} y^{2}+4.14578 y^{3}- \\
0.85 x y^{3}+1 . y^{4}+20.3972 x z+0.175 x^{2} z-0.829156 x^{3} z-6.01138 y z+ \\
6.1375 x y z+0.124373 x^{2} y z+0.0375 x^{3} y z-3.85 y^{2} z+ \\
1.15069 \times 10^{-16} x y^{2} z-0.25 x^{2} y^{2} z-1.8656 x z^{2}+1.1875 x^{2} z^{2}- \\
0.207289 x^{3} z^{2}-0.0625 x^{4} z^{2}+0.829156 y z^{2}-2.125 x y z^{2}+2.5 y^{2} z^{2}-0.625 x^{2} z^{3}
\end{array}\right)
$$

Now the graphical representation of the osculating plane equation $P_{1}(\vec{X})=0$ can be shown as follows: 


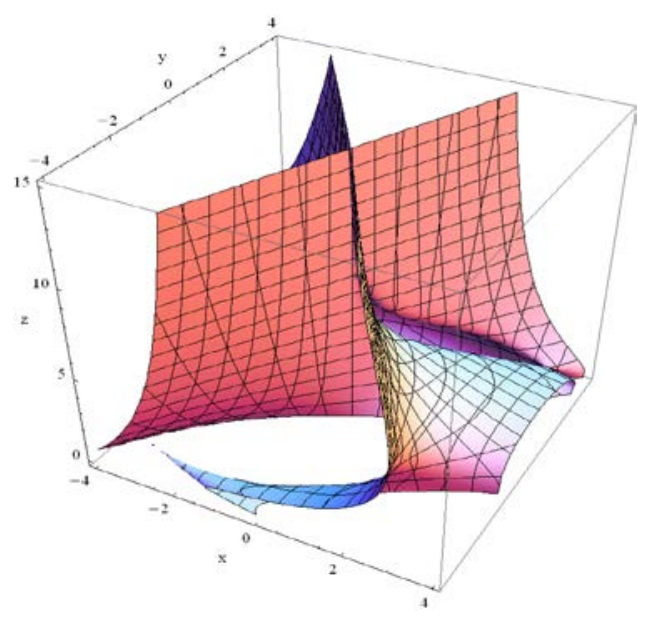

Fig.5. Graphical representation of the osculating plane equation corresponding to the fixed point $\left(\mathrm{x}_{1}, \mathrm{y}_{1}, \mathrm{z}_{1}\right)$.

Then we calculate the osculating plane equation for the fixed point $x_{2}=0, y_{2}=0, z_{2}=12$. We find the second osculating plane expression as the following

$$
P_{2}(\vec{X})=6.4\left(\begin{array}{l}
32.4 x^{2}-48.15 x y-2.4 x^{3} y+16.2 y^{2}+3.075 x^{2} y^{2}-0.025 x^{4} y^{2}-0.85 x y^{3}+1 . y^{4}- \\
9.45 x^{2} z+29.5125 x y z+0.0375 x^{3} y z-31.35 y^{2} z-0.25 x^{2} y^{2} z+8.0625 x^{2} z^{2}- \\
0.0625 x^{4} z^{2}-2.125 x y z^{2}+2.5 y^{2} z^{2}-0.625 x^{2} z^{3}
\end{array}\right)
$$

Now the graphical representation of the osculating plane equation $P_{2}(\vec{X})=0$ can be shown as follows:

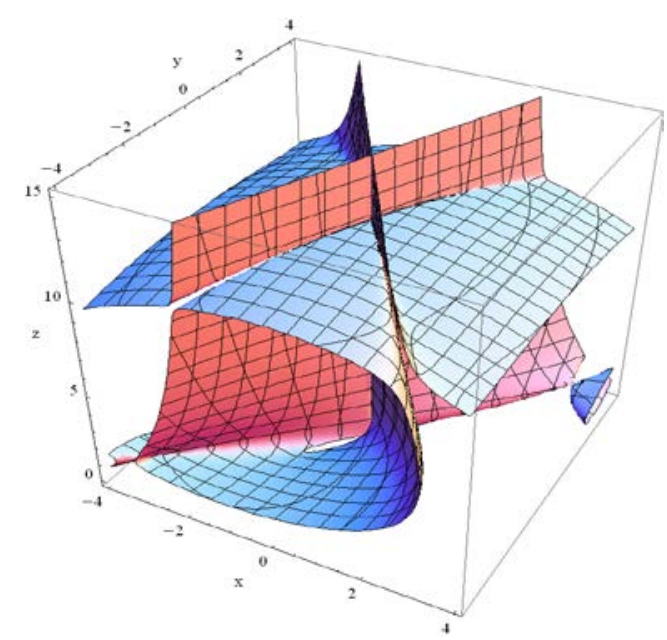

Fig.6. Graphical representation of the osculating plane equation corresponding to the fixed point $\left(\mathrm{x}_{2}, \mathrm{y}_{2}, \mathrm{z}_{2}\right)$. 
Similarly, we calculate the osculating plane equation for the fixed point $\mathrm{x}_{3}=3.31662479103554, \mathrm{y}_{3}=3.3166247903554, \mathrm{z}_{3}=1$. We find the third osculating plane expression as the following

$$
P_{3}(\vec{X})=6.4\left(\begin{array}{l}
-23.8797 x-0.6 x^{2}+47.2619 y+5.475 x y-3.15079 x^{2} y+0.35 x^{3} y-11.3 y^{2}+4.56036 x y^{2}+ \\
0.325 x^{2} y^{2}+0.0829156 x^{3} y^{2}-0.025 x^{4} y^{2}-4.14578 y^{3}-0.85 x y^{3}+1 . y^{4}-20.3972 x z+ \\
0.175 x^{2} z+0.829156 x^{3} z+6.01138 y z+6.1375 x y z-0.124373 x^{2} y z+0.0375 x^{3} y z-3.85 y^{2} z- \\
1.15069 \times 10^{-16} x y^{2} z-0.25 x^{2} y^{2} z+1.8656 x z^{2}+1.1875 x^{2} z^{2}+0.207289 x^{3} z^{2}-0.0625 x^{4} z^{2}- \\
0.829156 y z^{2}-2.125 x y z^{2}+2.5 y^{2} z^{2}-0.625 x^{2} z^{3}
\end{array}\right)
$$

Now the graphical representation of the osculating plane equation $P_{3}(\vec{X})=0$ can be shown as follows:

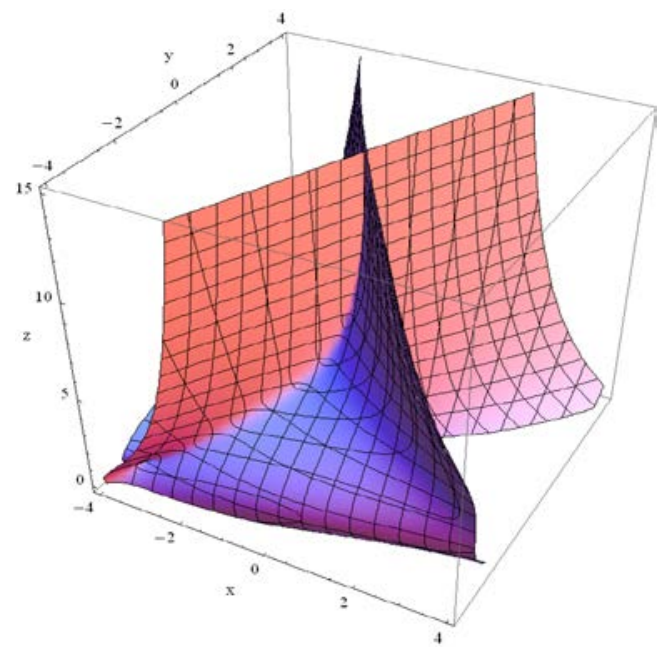

Fig.7. Graphical representation of the osculating plane equation corresponding to the fixed point $\left(\mathrm{x}_{3}, \mathrm{y}_{3}, \mathrm{z}_{3}\right)$.

Observation shows that, Fig.3(a) merges with Fig.5, Fig.6 and Fig 7, That means Theorem 3.4 holds.

\section{Stability Analysis of the Fixed Points Using Slow Invariant Manifold of the L-H model}

Fixed points of any dynamical system are the singular solutions of the flow curvature manifold and it's Lie derivative [14]. Thus fixed points may be deduced from the flow curvature manifold equation. The discriminant of the functional jacobian matrix associated with the dynamical systems may be deduced from the flow curvature manifold which contains good information about the dynamics of the system.

The following expression represents the first row vector of the Hessian matrix of the flow curvature manifold at the point $\vec{X}=(x, y, z)^{t}$. 


$$
\begin{aligned}
& \left\{-4202.5+98.304 y^{4}+x^{3} y(-122.88-7.68 z)+1631.23 z-413.952 z^{2}+179.2 z^{3}-12.8 z^{4}+\right. \\
& x^{4}\left(7.68 y^{2}+19.2 z^{2}\right)+y^{2}\left(-1139.71-367.616 z+217.6 z^{2}\right)+x^{2}\left(442.368+y^{2}(55.296-61.44 z)-\right. \\
& \left.866.304 z+138.24 z^{2}-153.6 z^{3}\right)+x y\left(1870.85+1032.19 z-399.36 z^{2}+115.2 z^{3}+y^{2}(-168.96+46.08 z)\right), \\
& 4787.71+3.072 x^{5} y-153.6 y^{4}+x^{3} y(36.864-40.96 z)+x^{4}(-30.72-1.92 z)-1142.4 z+292.352 z^{2}- \\
& 19.2 z^{3}+y^{2}\left(586.752-18.432 z-230.4 z^{2}\right)+x y\left(-2279.42+393.216 y^{2}-735.232 z+435.2 z^{2}\right)+ \\
& x^{2}\left(935.424+516.096 z-199.68 z^{2}+57.6 z^{3}+y^{2}(-253.44+69.12 z)\right),-1.92 x^{4} y+7.68 x^{5} z+ \\
& x^{3}\left(-288.768-20.48 y^{2}+92.16 z-153.6 z^{2}\right)+y\left(-1142.4+y^{2}(-6.144-153.6 z)+584.704 z-57.6 z^{2}\right)+ \\
& \left.x^{2} y\left(516.096+23.04 y^{2}-399.36 z+172.8 z^{2}\right)+x\left(1631.23-827.904 z+537.6 z^{2}-51.2 z^{3}+y^{2}(-367.616+435.2 z)\right)\right\}
\end{aligned}
$$

The following expression represents the second row vector of the Hessian matrix of the flow curvature manifold at the point $\vec{X}$.

$$
\begin{aligned}
& \left\{4787.71+3.072 x^{5} y-153.6 y^{4}+x^{3} y(36.864-40.96 z)+x^{4}(-30.72-1.92 z)-1142.4 z+\right. \\
& 292.352 z^{2}-19.2 z^{3}+y^{2}\left(586.752-18.432 z-230.4 z^{2}\right)+x y\left(-2279.42+393.216 y^{2}-\right. \\
& \left.735.232 z+435.2 z^{2}\right)+x^{2}\left(935.424+516.096 z-199.68 z^{2}+57.6 z^{3}+y^{2}(-253.44+69.12 z)\right), \\
& -7114.75+0.512 x^{6}+x^{4}(9.216-10.24 z)+2104.32 z-740.352 z^{2}+51.2 z^{3}+x^{3} y(-168.96+46.08 z)+ \\
& y^{2}(2211.84+491.52 z)+x y\left(1173.5-614.4 y^{2}-36.864 z-460.8 z^{2}\right)+x^{2}\left(-1139.71+589.824 y^{2}-\right. \\
& \left.367.616 z+217.6 z^{2}\right),-0.384 x^{5}-10.24 x^{4} y+x^{2} y(-367.616+435.2 z)+x\left(-1142.4+y^{2}(-18.432-460.8 z)+\right. \\
& \left.\left.584.704 z-57.6 z^{2}\right)+x^{3}\left(172.032+23.04 y^{2}-133.12 z+57.6 z^{2}\right)+y\left(2104.32+163.84 y^{2}-1480.7 z+153.6 z^{2}\right)\right\}
\end{aligned}
$$

The following expression represents the third row vector of the Hessian matrix of the flow curvature manifold at the point $\vec{X}$.

$$
\begin{aligned}
& \left\{-1.92 x^{4} y+7.68 x^{5} z+x^{3}\left(-288.768-20.48 y^{2}+92.16 z-153.6 z^{2}\right)+y\left(-1142.4+y^{2}(-6.144-153.6 z)+\right.\right. \\
& \left.584.704 z-57.6 z^{2}\right)+x^{2} y\left(516.096+23.04 y^{2}-399.36 z+172.8 z^{2}\right)+x\left(1631.23-827.904 z+537.6 z^{2}-\right. \\
& \left.51.2 z^{3}+y^{2}(-367.616+435.2 z)\right),-0.384 x^{5}-10.24 x^{4} y+x^{2} y(-367.616+435.2 z)+x(-1142.4+ \\
& \left.y^{2}(-18.432-460.8 z)+584.704 z-57.6 z^{2}\right)+x^{3}\left(172.032+23.04 y^{2}-133.12 z+57.6 z^{2}\right)+ \\
& y\left(2104.32+163.84 y^{2}-1480.7 z+153.6 z^{2}\right), 1.28 x^{6}+x y\left(584.704-153.6 y^{2}-115.2 z\right)+ \\
& x^{4}(23.04-76.8 z)+x^{3} y(-133.12+115.2 z)+y^{2}(-740.352+153.6 z)+ \\
& \left.x^{2}\left(-413.952+217.6 y^{2}+537.6 z-76.8 z^{2}\right)\right\}
\end{aligned}
$$

By combining these three row vectors we find the complete Hessian matrix. The determinant of this Hessian matrix is denoted by $H_{\psi(\vec{X})}$. The relative Hessian of the flow curvature manifold $\psi(\vec{X})$ can be defined as follows:

$$
\hat{H}_{\psi(\vec{X})}=\frac{H_{\psi(\vec{X})}}{\psi(\vec{X})}
$$

We now calculate the relative Hessian at the point $\left(x_{1}+\varepsilon, y_{1}+\varepsilon, z_{1}+\varepsilon\right)$ and get the following expression 


$$
\begin{aligned}
& \left(3.64235 \times 10^{-33}+\varepsilon\left(-3.71533 \times 10^{-18}+\varepsilon\left(-0.000552965+\varepsilon\left(-1.53476 \times 10^{11}+\varepsilon\left(5.30899 \times 10^{11}+\right.\right.\right.\right.\right. \\
& \varepsilon\left(-8.87961 \times 10^{11}+\varepsilon\left(9.18391 \times 10^{11}+\varepsilon\left(-5.94088 \times 10^{11}+\varepsilon\left(2.21072 \times 10^{11}+\varepsilon\left(-2.68341 \times 10^{10}+\right.\right.\right.\right.\right. \\
& \varepsilon\left(-1.64071 \times 10^{10}+\varepsilon\left(1.03109 \times 10^{10}+\varepsilon\left(-2.97524 \times 10^{9}+\varepsilon\left(5.26477 \times 10^{8}+\varepsilon\left(-5.95009 \times 10^{7}+\right.\right.\right.\right.\right. \\
& \left.\left.\left.\left.\left.\left.\left.\left.\left.\left.\left.\left.\left.\left.\left.\varepsilon\left(4.16963 \times 10^{6}+\varepsilon(-167288 .+(3408.12-27.5251 \varepsilon) \varepsilon)\right)\right)\right)\right)\right)\right)\right)\right)\right)\right)\right)\right)\right)\right)\right)\right) /\left(8.31538 \times 10^{-} 12+\right. \\
& \varepsilon\left(7.79567 \times 10^{-} 12+\varepsilon\left(-2.18279 \times 10^{-11}+\varepsilon(1844.25+\varepsilon(-1388.47+\varepsilon(288.525+\varepsilon(13.4831+\right.\right. \\
& \varepsilon(-10.795+1 . \varepsilon))))))))
\end{aligned}
$$

By considering $\varepsilon \rightarrow 0$, we get the positive value of the above expression and which is $4.38026 \times 10^{-22}$. Now according to theorem 3.5, the fixed point $\left(\mathrm{x}_{1}, \mathrm{y}_{1}, \mathrm{z}_{1}\right)$ is a saddle-node. Then we calculate the relative Hessian at the point $\left(x_{2}+\varepsilon, y_{2}+\varepsilon, z_{2}+\varepsilon\right)$ and get the following expression

$$
\begin{aligned}
& \left(0 .+\varepsilon^{2}\left(0.00224639+\varepsilon\left(-2.59169 \times 10^{12}+\varepsilon\left(-3.29468 \times 10^{12}+\varepsilon\left(-1.62118 \times 10^{12}+\varepsilon\left(9.43401 \times 10^{12}+\right.\right.\right.\right.\right.\right. \\
& \varepsilon\left(4.36915 \times 10^{12}+\varepsilon\left(4.53163 \times 10^{12}+\varepsilon\left(2.3954 \times 10^{12}+\varepsilon\left(6.03654 \times 10^{11}+\varepsilon\left(7.94268 \times 10^{10}+\right.\right.\right.\right.\right. \\
& \varepsilon\left(4.72054 \times 10^{9}+\varepsilon\left(-7.13001 \times 10^{7}+\varepsilon\left(-2.74377 \times 10^{7}+\varepsilon\left(-1.22338 \times 10^{6}+\varepsilon(12093.5+\right.\right.\right.\right. \\
& (1462.76-27.5251 \varepsilon) \varepsilon)))))))))))))))) /\left(\varepsilon ^ { 2 } \left(-1.9749 \times 10^{-11}+\varepsilon(-9904.71+\varepsilon(5526.71+\right.\right. \\
& \varepsilon(2178 .+\varepsilon(343.714+\varepsilon(26.7143+1 . \varepsilon)))))))
\end{aligned}
$$

By considering $\varepsilon \rightarrow 0$, we get the negative value of the above expression and which is $-1.13747 \times 10^{8}$. Now according to theorem 3.5, the fixed point $\left(\mathrm{x}_{2}, \mathrm{y}_{2}, \mathrm{z}_{2}\right)$ is a saddle-focus or center. Similarly, we calculate the relative Hessian at the point $\left(x_{3}+\varepsilon, y_{3}+\varepsilon, z_{3}+\varepsilon\right)$ and get the following expression

$$
\begin{aligned}
& \left(3.15053 \times 10^{-33}+\varepsilon\left(3.55972 \times 10^{-18}+\varepsilon\left(0.000208321+\varepsilon\left(-4.44938 \times 10^{11}+\varepsilon\left(-1.79251 \times 10^{12}+\right.\right.\right.\right.\right. \\
& \varepsilon\left(-2.97502 \times 10^{12}+\varepsilon\left(-2.69281 \times 10^{12}+\varepsilon\left(-1.51324 \times 10^{12}+\varepsilon\left(-5.66076 \times 10^{11}+\varepsilon\left(-1.47005 \times 10^{11}+\right.\right.\right.\right.\right. \\
& \varepsilon\left(-2.74232 \times 10^{10}+\varepsilon\left(-3.83535 \times 10^{9}+\varepsilon\left(-4.22314 \times 10^{8}+\varepsilon\left(-3.57007 \times 10^{7}+\varepsilon\left(-1.58142 * 10^{6}+\right.\right.\right.\right.\right. \\
& \varepsilon(86197.8+\varepsilon(15605.6+(382.487-27.5251 \varepsilon) \varepsilon))))))))))))))))) /\left(8.31538 * 10^{-} 12+\varepsilon\left(2.91038 * 10^{-11}+\right.\right. \\
& \left.\left.\varepsilon\left(2.39067 * 10^{-} 11+\varepsilon(5346.61+\varepsilon(5115.9+\varepsilon(1914.62+\varepsilon(356.517+\varepsilon(32.795+1 . \varepsilon)))))\right)\right)\right)
\end{aligned}
$$

By considering $\varepsilon \rightarrow 0$, we get the positive value of the above expression and which is $3.7888 \times 10^{-22}$. Now according to theorem 3.5 , the fixed point $\left(\mathrm{x}_{3}, \mathrm{y}_{3}, \mathrm{z}_{3}\right)$ is a saddle-node.

\section{Conclusion}

In this work, we applied a newly developed strategy which reflects the applications of differential geometry in the dynamical system called flow curvature method to the L-H model. We determined the curvature of the trajectory curve or the curvature of the flow analytically called flow curvature manifold by estimating the solution or trajectory curve of the dynamical system as a curve in Euclidean space. Since this manifold comprises the time derivatives of the velocity vector field and hence it receives knowledge about the dynamics of the corresponding system. L-H model is a well-known problem as a nonlinear optical slow-fast dynamical system. In this manuscript, we analyzed the slow invariant manifold of the three-dimensional L-H model. We used flow curvature method to determine the analytical equation of the slow invariant manifold of the model. 
We performed a numerical simulation of the L-H model by using Explicit Runge-Kutta method. We also used Darboux invariance theorem to show the invariance of the slow manifold. We also determined three osculating plane equations for three different fixed points and found a good correspondence between flow curvature manifold and osculating plane equations. Then we performed fixed point stability analysis through the Hessian of the flow curvature manifold.

\section{Acknowledgements}

The author wish to thank Dr. Md. Showkat Ali for his guidance and supervision during the research period and also thank the anonymous reviewers for their valuable comments and suggestions to improve the quality of the paper.

\section{References}

[1] Andronov, A.A., Chaikin, S.E (1937). Plane Theory of Oscillators, I, Moscow.

[2] Tikhonov, A.N. (1948). On the dependence of solutions of differential equations on a small parameter, Mat. Sbornik N. S., 31:575-586.

[3] Levinson, N., (1949). A second-order differential equation with singular solutions, Ann. Math, 50:127153.

[4] Fenichel, N. (1971). Persistence and smoothness of invariant manifolds for flows, Indiana Univ. Math. J, 21:193-225.

[5] Fenichel, N. (1974). Asymptotic stability with rate conditions, Indiana Univ. Math. J, 23:1109-1137.

[6] Fenichel, N. (1977). Asymptotic stability with rate conditions II, Indiana Univ. Math. J, 26:81-93.

[7] Fenichel, N. (1979). Geometric singular perturbation theory for ordinary differential equations, J. Differ. Equ., 31: 53-98.

[8] Wasow, W.R. (1965). Asymptotic Expansions for Ordinary Differential Equations, Wiley-Interscience, New York.

[9] Cole, J.D. (1968). Perturbation Methods in Applied Mathematics, Blaisdell, Waltham.

[10] O’Malley, R.E. (1974). Introduction to Singular Perturbations, Academic Press, New York.

[11] O’Malley, R.E. (1991). Singular Perturbations Methods for Ordinary Differential Equations, Springer, New York.

[12] Ginoux, J.M. and Rossetto, B. (2006). Differential geometry and mechanics applications to chaotic dynamical systems, Int. J. Bifurc. Chaos, 4(16): 887-910.

[13] Ginoux, J.M., Rossetto, B. and Chua, L.O. (2008). Slow invariant manifolds as curvature of the flow of dynamical systems, Int. J. Bifurc. Chaos, 11(18): 3409-3430.

[14] Ginoux, J.M. (2009). Differential geometry applied to dynamical systems, In: World Scientific Series on Nonlinear Science, Series A, 66, World Scientific, Singapore.

[15] Ginoux, J.M.and Llibre, J. (2011). The flow curvature method applied to canard explosion, J. Phys. A Math. Theor., 44: 465203.

[16] Ginoux, J.M., Llibre, J. and Chua, L.O. (2013). Canards from Chua’s circuit, Int. J. Bifurc. Chaos, 23(4): 1330010.

[17] Ginoux, J. M., \& Rossetto, B. (2014). Slow invariant manifold of heartbeat model, arXiv preprint arXiv:1408.4988.

[18] Ginoux, J. M. (2014). The slow invariant manifold of the Lorenz-Krishnamurthy model, Qualitative theory of dynamical systems, 13(1): 19-37.

[19] Rossetto, B., Lenzini, T., Ramdani, S. \& Suchey, G. (1998). Slow-fast autonomous dynamical systems, Int. J. Bifurcation and Chaos, 8(11): 2135-2145. 
[20] Ramdani, S. (2000). Slow manifolds of some chaotic systems with applications to laser systems, Int. J of bifurcation and Chaos, 10 (12): 2729-2744.

[21] Cai, G., Tian, L., \& Huang, J. (2006). Slow manifolds of Lorenz-Haken system and its application, International Journal of Nonlinear Science, 1(2): 93-104.

[22] Haken, H. (1975). Analogy between higher instabilities in fluids and lasers, Phys. Lett. A, 53(1):77-78.

[23] Schlomiuk D. (1999). Elementary first integrals of differential equations and invariant algebraic curves , Expositiones Mathematicae, 11: 433-454.

[24] Llibre J . \& Medrado J. C. (2007). On the invariant hyperplanes for d-dimensional polynomial vector fields , J. Phys. A. Math. Theor., 40 : 8385-8391.

\section{Authors' Profiles}

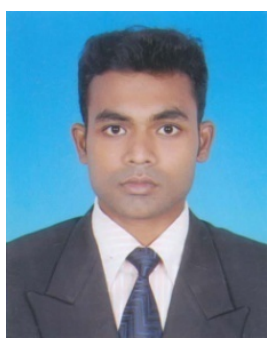

A. K. M. Nazimuddin received his B. S. (Hons) degree in Mathematics and M. S. in Applied Mathematics from the University of Dhaka. He is working as a Senior Lecturer in the Department of Mathematical and Physical Sciences, East West University, Dhaka, Bangladesh. His research interest is on Dynamical Systems, Riemannian Geometry, Symplectic Geometry and Contact Geometry.

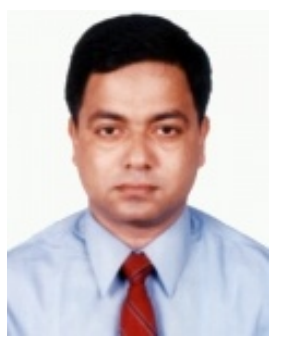

Md. Showkat Ali received his PhD from the University of Glasgow, Glasgow, UK. He received his B. S. (Hons) degree in Mathematics and M. S. in Applied Mathematics from the University of Dhaka, Bangladesh. He is working as a Professor in the Department of Applied Mathematics, University of Dhaka. His research interest is on Differential Geometry and Dynamical Systems. He has published about fifty research articles in this field in various reputed national and international journals.

How to cite this paper: A. K. M. Nazimuddin, Md. Showkat Ali," Application of the Flow Curvature Method in Lorenz-Haken Model ", International Journal of Mathematical Sciences and Computing (IJMSC), Vol.6, No.1, pp.33-48, 2020. DOI: 10.5815/ijmsc.2020.01.04. 